Running head: Feeding schedule in male Barn Owls

\title{
Diel feeding strategy during breeding in male Barn Owls (Tyto alba)
}

Joël M. Durant ${ }^{1 *}$, Dag Ø. Hjermann ${ }^{2}$ and Yves Handrich ${ }^{3}$

${ }^{1}$ Centre for Ecological and Evolutionary Synthesis (CEES), Department of Biosciences, University of Oslo, PO Box 1066 Blindern, N-0316 Oslo, Norway

2 Norwegian Institute for Water Research (NIVA), Gaustadalléen 21, N-0349 Oslo, Norway

${ }^{3}$ Institut Pluridisciplinaire Hubert Curien (IPHC), Département Ecologie, Physiologie et Ethologie (DEPE), Centre National de la Recherche Scientifique, 23 rue Becquerel, F-67087 Strasbourg Cedex 02, France

* Corresponding author: J.M. Durant, phone: +47-22854795; fax: +47-22854001 (Attn.: Durant); e-mail joel.durant@ibv.uio.no 


\section{Abstract}

2 During reproduction birds are confronted with two requirements: building up their energetic

3 reserves during the activity period, and provisioning the nest. Storing reserves imposes a higher

4 flight cost and a lower hunting efficiency. This conflict is accentuated in species such as the Barn

5 Owl (Tyto alba), where the nest food supply is entirely taken in charge by the male during a long

6 period (ca 37 days). We tested the prediction that Barn Owl males were postponing their meal to

7 the end of the night in order to fly with a low body mass. Nocturnal changes in body mass (i.e.,

8 estimates of feeding events) were monitored remotely on six free ranging barn owl pairs nesting

9 in eastern France using an automated weighing system. Male Barn Owls were gaining the more

10 weight at the end of the night, supporting the prediction that they make their biggest meal after

11 the nest provisioning activities hunting period that is taking place at the beginning of the night.

12

13 Key words: Raptors - Food intake - Body mass - Reproduction - Behaviour - Diet 


\section{Introduction}

During the breeding season, there is a trade-off between allocating resources to adult survival or to production of offspring (Roulin, 2001; Stearns, 1992; Williams, 1966). While brooding, many female raptors rely exclusively on food provisioning from males. For males, the energy cost of hunting is then a very important factor affecting the trade-off between survival and reproduction.

To the same extent as the effect of territory quality and variations in weather (Hakkarainen et al., 1997), and the duration of the female food dependence when it applies (Newton, 1979), the intrinsic cost of flight is an important factor to estimate the cost of reproduction in male raptors (Norberg, 1996). One of the main elements affecting the energy cost of flight is the mass that the bird has to lift (Norberg, 1996). Within the same species and for a given wingspan, lighter individuals have a lower cost for flight (Norberg, 1996). Indeed, the reduction in body mass has been interpreted as a mechanism to reduce wing loading and optimize foraging (Sanz and Moreno, 1995). Within a day, the body mass can also change greatly as a result of the timing of feeding events (Durant, 2000). As a consequence, the timing of the feeding events (and its impact on body mass) in relationship to the foraging activity may be an important factor in the cost of flight

The Barn Owl, Tyto alba (Scopoli, 1769), is a monogamous species that commonly breed in urban area in Eastern France. Barn Owls catch prey up to $2 \mathrm{~km}$ from the nest site with the majority within $1 \mathrm{~km}$ (Taylor, 1994). Breeding in houses (garrets, roofs and church’s towers) Barn Owls have to fly over the surrounding houses to reach their nest from the hunting ground carrying their prey, which may be very heavy (e.g. Arvicola terrestris of $120 \mathrm{~g}$ compared to a Barn Owl mass of ca 300 g; Roulin, 2004) .The female Barn Owl usually lays clutches of 4-7 
eggs depending on food availability (Durant, 2000; Durant et al., 2010; Muller, 1991). The female starts incubation after laying the $1^{\text {st }}$ egg of the clutch and stays in the nest continuously, thus depending on the male for her food supply (Bunn et al., 1982; Durant et al., 2004; Shawyer, 1994; Taylor, 1994) until she reinitiates foraging ca two weeks after hatching of the first egg (Durant, 2002; Durant et al., 2004; Taylor, 1994). In some cases of late offspring desertion by the female, the male can even take care of the brood alone (Roulin, 2002). Consequently, the male Barn Owl is confronted with more than one month (ca 37 days, Durant et al., 2010) of intense hunting to provide prey to his mate and later on to the nestlings. Indeed, during rearing the male can deliver to the nest up to 11 prey of vole size per night, which represents a cumulative mass of more than 80\% of its body mass (ca 290 g; Durant et al., 2004). Durant et al (2004) suggested that the reinitiation of foraging for the female was linked to the male food provisioning that no longer matches nestling food requirements. Knowing that the survival of the last hatched chicks depends on the female care and on her staying in nest to feed them (Durant et al., 2004), the hunting capacity of the male has a strong influence on the reproductive success in this species.

The aim of the present study is to determine the feeding time of breeding males during the active period (i.e., night). Our hypothesis is that due to the advantage of a lower body mass for foraging (lower metabolic power required for flight, Norberg, 1996), males take their main meal at the end of their period of activity (Rijnsdorp et al., 1981). If such an advantage exists, there should be an energy economy associated to this behaviour. As also implicit to this hypothesis, assuming that the males are fasting during their day-time rest we make a second hypothesis that they are starting their hunting period by a meal. Following our first hypothesis such meal should be smaller than the last one. 
The first step to verify our hypothesis is to know when males are taking their meal and the

60

size of it. Recording eating events is notoriously difficult for nocturnal raptors, which are sensitive to disturbance during breeding. We therefore investigated the timing of the body mass gains during the day assuming that each mass gain is due to a feeding event. We report here the changes in body mass of six free ranging male barn owls during reproduction. Data were obtained using nest boxes equipped with an electronic balance and a video camera (Durant, 2000; Durant, 2002; Durant et al., 2004). Body mass and activity were thus automatically recorded when males visit the nest (the male was weighed once when it enters and once when it leaves the nest). With this system and considering the frequency of nest visits, it was possible to precisely quantify the body mass changes and calculate the mass gains. Data thus collected were analysed using nonparametric quantile regression techniques on the timing of the heaviest meal of each night. The size of the meal (body mass change) may, however, depend on the size of the available prey. Indeed, while a small mammal specialist, Barn Owl is also an opportunist depending of the local availability within its hunting territory (Mikkola, 1983; Shawyer, 1994). Our system makes it possible to identify the prey delivered by the male and estimate their mass. To ascertain that the heaviest prey were available during the whole activity period, and not only at the end of it, we looked at the pattern of the prey mass delivered to the nest throughout the activity period. To ascertain that prey availability was similar between males we investigated the prey spectrum delivered to the nest. We calculated the Schoener's index on the prey delivered to the nest assuming that they represent the prey spectrum available (Schoener, 1968, Mysterud, 2000).

Finally, the second step to verify our hypothesis is to ascertain that there is a benefit to such eating behaviour, i.e., energy sparing by eating at the end of the night. We estimated the metabolic power required for flight applied to three scenarios and compared the energy costs between them: 
1 - the male eats his meal anytime during the night; 2 - the male eats his meal during two bouts, one prey item at the beginning and one prey item at the end of the night; and 3 - the male eats his meal at the end of the night only.

\section{Material and Methods}

The study was conducted in Alsace, eastern France $\left(48^{\circ} 20^{\prime} \mathrm{N}, 7^{\circ} 45^{\prime} \mathrm{E}\right)$ on six pairs of Barn Owl (hybrids of Tyto alba alba and Tyto a. guttata). The clutches were on average composed of 4.5 eggs (3, 5, 3, 6, 6, 4) which led to about 3.3 fledglings (3, 4, 2, 4, 4, 3). We defined an "owl-day” as the period ranging from 1600 to 1559 hours the following day in order to group the foraging events occurring before and after midnight within the same day. Hereafter “day” refers to an “owl-day”. Days were numbered from the hatching of the last egg (day 0) in order to synchronize the broods. Timing was considered in a day scale ( 1 hour $=0.0417$ day). Nest monitoring begun at the latest 27 days before the laying of the first egg (range 27-39 days) and finished $58 \pm 8$ ( \pm SD) days (range 43-95) after hatching of the first egg. Moult occurred for male Barn Owls mostly after the young have fledged (July-October, Taylor 1994) and was not affecting the study.

\section{Monitoring design}

Data on mass and behaviour were obtained using nest boxes equipped with an electronic balance located in a 1 meter long access corridor and an infrared video camera (Durant, 2002; Durant et al., 2004, 2010). The system was installed in wooden nest boxes regularly used by breeding barn owls. The weighing and the time of passage were recorded on a computer and the behaviour on a tape recorder, both devices synchronized. 

inside the nest room was monitored by video (24h day ${ }^{-1}$ recording on tape) which allows us to identify differences in plumage between the male and the female during sex specific behavior (copulation, egg laying, and egg incubation). Sex identification by video was confirmed by mass recording. Indeed, during breeding, the female Barn Owl on average weights 33\% more than the male (respectively 387 g and 290g; Durant et al., 2004). During the studied period the males have entered the nest on average $7.0 \pm 3.3$ times/day while the females did it on average $5.2 \pm 3.0$ times/day. However, the pattern of visits is not the same over time (see Fig. 1 and Durant et al. 112 2010).

Each time a bird walked across the weighing platform the body mass was recorded with a

114 frequency of 34 measures per sec. Due to the access corridor configuration, it takes several seconds for the bird to cross it (5.65 $\pm 0.10( \pm \mathrm{SE})$ s (range 0.09-42.48), $N=9375)$, body mass was recorded on average 115 times during a passage. An average adult body mass for each passage over the weighing platform was thus calculated using custom-made software (J. Lage 118 Jensen Software Systems). 82\% after hatching (Durant et al., 2010). Males always leaved the nest without prey. The weighing recorded at the exit corresponds to the male mass (hence weighing when the male cross the balance without bearing a prey). The body mass gain (dBM) is the difference between two consecutive male body mass measurements (exits). We assumed that a $\mathrm{dBM}$ increase was due to food intake (overlooking the loss of mass due to pellet ejection and faeces). For the analysis we 125 only retained $\mathrm{dBM}$ above $+10 \mathrm{~g}$ to take into account for the accuracy of the balance. The retained dBMs were then associated to the time of the second exit (ranging from $0-1$ with $0=1600$ ) for the 
analysis. Figure 2 presents the data for the six males followed (see data in Electronic

Supplementary Material, ESM, Fig. 4). For each owl and each day we extracted the maximum body mass gain (max dBM). Further details on methods can be found in Durant (2002) and Durant (2004).

Video recording allowed prey to be identified when delivered by the male to the nest box. We classified the prey into 5 categories: "Vole" for prey of the genera Microtus; "Field mouse" for genera Apodemus and Mus; "Arvicola" for Arvicola; "Shrew" for the genera Crocidura and Sorex; and "Others" for birds, amphibia, insects and bats (for a total of 90, 103, 25, 21, and 145 items per category respectively). The mass of each prey was calculated as the difference between the mass measured when the male entered with a prey and the mass measured when he exited the nest without the prey (see above).

To ascertain that the males have access to similar prey, we have calculated the diet overlap O using Schoener’s index (Schoener, 1968; Mysterud, 2000):

$$
\mathrm{Ojk}=1-0.5 * \Sigma|\mathrm{Pij}-\mathrm{Pik}|
$$

where Ojk is the overlap between the males $\mathrm{j}$ and $\mathrm{k}$; Pij is the proportion of prey species/group i brought to nest by male j out of the total number of prey; and Pik is the proportion of prey species/group i brought to nest by male k out of the total number of prey. Overlap in prey spectrum brought to nest between males $\mathrm{j}$ and $\mathrm{k}$ is complete when $\mathrm{Ojk}=1 \mathrm{and}$ is absent when Ojk $=0$ (Mysterud, 2000).

\section{Density plots}

48 Density plot is a convenient method to visualize our data. With such plots it is possible to visualize when during a day an event (mass change, behaviour...) is occurring the most frequently 
and to follow this during the time period studied. We built density plots for both $\mathrm{dBM}$ and prey mass (Fig. 3 a and b, see also ESM Fig. 6). For each day and each male, we extracted both the time of the night when the bigger dBM (heaviest meal of the day) occurred and when the heaviest prey was brought to the nest. Using the $k d e 2 d$ function from the R package MASS we obtained a two-dimensional kernel density estimate (the $\mathrm{x}$-axis being days and the $\mathrm{y}$-axis time of the day) and plotted these densities. The higher the density (i.e., more frequent is the event) the lighter is the colour (white for Fig.3 and yellow for ESM Fig. 6).

\section{Statistical analyses}

Statistical analysis was conducted using the statistical package R 2.14.1 (R Development Core Team, 2011). We related the change in body mass gain (dBM) with the time of the night using a nonparametric quantile regression using the function $r q$ implemented in the quantreg library (Cade and Noon, 2003) and the function bs implemented in the splines library. The essence of quantile regression is that the usual single-mean function estimated in a linear or nonlinear model is replaced with a family of functions across a selected subset of quantiles on the interval $[0,1]$. We tested the individual effect by adding male as factor in a Generalized Additive Model (GAM) formulation as implemented in the mgcv library (Wood and Augustin, 2002). The individual effect was not significant (mean $\mathrm{p}>0.648$, range 0.19-0.94) and did not add to the model (Generalized Cross Validation criterium 2.847 vs 2.819 for the simpler model, see ESM Fig. 5). Day-to-day changes in male body mass were estimated by linear mixed effects models using the function Imer implemented in the Ime4 library with the male entered as random variables. 
For each male, we estimated the metabolic power required for flight using the formula

173

174

175

176

177

178

179

180

181

182

183

184

185

186

187

188

189

190

191

192

193

.

$\mathrm{P}(\mathrm{W})=57.3 * \mathrm{BM}^{0.813}(\mathrm{~kg})($ Norberg, 1996) and the average body mass (BM). BM and $\mathrm{P}$ were

modified by the meal size eaten during the night. Meal size was estimated through the

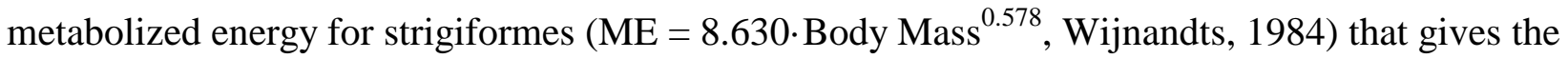

energy required by bird per day. This daily energy requirement was transformed in a mass of prey

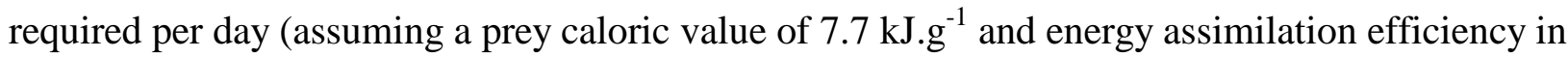

Barn Owl of 72.3 \%; Durant et al. 2000). Following what was found for Long-eared Owl Asio

otus (Wijnandts, 1984), we assumed that Barn Owl males fly 3h per 24h (unevenly distributed, with $2 \mathrm{~h}$ at the beginning and $1 \mathrm{~h}$ at the end of the hunting period). We applied 3 scenarios: 1 - the male eats his meal anytime during the night; 2 - the male eats his meal during two bouts, one prey item at the beginning and one prey item at the end of the night; and 3 - the male eats his meal at the end of the night. The energy cost of flight was then compared between the different scenarios using a paired t-test for each male after running 1000 replicates.

\section{Results}

The male body mass (BM) was on average 302.1 $\pm 0.8 \mathrm{~g}$ (range 287-310) during the reproduction. There was a significant decrease of about 0.15 g per day (lmer: $\mathrm{p}<0.001$ ) during the studied period. However, this decrease corresponded to less than $5 \%$ of the body mass only (c. -10 g over the 65 days of the study).

The average body mass gain (dBM) per weighing was $20.6 \pm 0.4$ g (range 19.7 - 21.9) with a maximum mass gain ranging from 49.3 to $98.2 \mathrm{~g}$ (see ESM Fig. 4). Assuming that the body mass gain corresponds to feeding, the size of meals was not uniformly distributed along the 


6

night (white in Fig. 2 and ESM Fig. 5). Males seemed to make a small meal at the beginning of the night, then a big meal at the end of the night (Fig. 3a).

The prey delivery spectrum was similar between males (Durant et al., 2010). On average overlap of prey delivery between males was $\mathrm{O}=0.988 \pm 0.017$. If we consider that when overlap $\mathrm{O}>2 * \mathrm{SD}$ of the diet overlap between males was full and highly significant. The heaviest prey were delivered to the nest at the beginning of the night (Fig. 3b), independently of the prey category considered (ESM Fig. 6).

The time and the number of meals have a significant but very small effect on the flight cost. Compared to scenario 1 (1 meal anytime in the night), scenario 2 (1 meal at the beginning and 1 meal at the end of the night) was significantly more costly $0.12 \pm 0.01 \mathrm{~W}(\mathrm{t}=-13.64, \mathrm{p}<$ 0.001). Compared to scenario 1 , scenario 3 (1 meal at the end of the foraging period) was significantly less costly $-1.59 \pm 0.02 \mathrm{~W}(\mathrm{t}=110.88, \mathrm{p}<0.001)$.

We show that during reproduction male Barn Owls, while eating throughout the night (Fig. 2), tend to take their main meals at the end of the night-time and that this pattern is true during the whole reproduction. Using radio-tagged males, Taylor (1994) suggested that male Barn Owls start to consume prey after catching six to eight items, and that they caught all their prey during the first 3 hours or so of hunting. Indeed, parent Barn Owls provision their brood mainly during the first part of the night (Michelat and Giraudoux, 1992; Ritter and Görner, 1975; Roulin, 2001) and thus certainly satisfy their own needs during the second part (Taylor, 1994). However, in our study we show that while the heaviest meal was taken at the end of the night, males were also eating at the beginning of the night. In addition, males brought prey to the nest during the whole 
night (Fig. 3b). This behaviour would be coherent with them hunting all their prey at the beginning of the night (Taylor, 1994) if the Barn Owl males caches prey outside the nest, as done by the common Kestrel Falco tinnunculus (Masman et al., 1986). Noteworthy is the observation that the males usually brought the heaviest prey to the nest at the beginning of the night. This indicates that male Barn Owls have the possibility to eat bigger meals at the beginning of the hunting period than they actually did and were not constrained by the size of the available prey. Barn Owls being opportunist in their prey choice, this result also indicates that the males caught the heaviest prey at the beginning of the night and possibly caches food outside the nest. That male Barn Owls capture and deliver the heaviest prey to the nest at the beginning of the hunting period could also be an answer to the hunger of the nestlings/female at this time (end of the daytime rest). While this may be the case when the food requirement is at its maximum (ca 30 days after the hatching of the first egg; Durant et al., 2004 ), prior to it we often observed remaining prey in the nest chamber before the delivery of its first prey by the male (Baudvin, 1980). Note that before 15 days of age, nestling Barn Owls cannot swallow whole prey and that food is dispatched to them by the female throughout the day. Finally, while the heaviest prey were delivered to the nest at the beginning of the night, the delivery frequency was not higher during this period as would be expected if there was an higher incentive to the male to deliver prey concomitant to the high hunting possibilities at sun-down (see below).

Barn Owls do not have a lot of body reserves (Handrich et al., 1993; Thouzeau et al., 1999). This could be a reason why they eat throughout the night (Fig. 2). However eating big meals before the high activity period could be energetically expensive (see below). As with passerines, Barn Owls may be confronted to the conflict of assimilating a meal during the activity period and the mass-dependent cost of flying (Thomas, 2000; Witter and Cuthill, 1993). The 
tendency to postpone feeding to the end of the nest provisioning activity can be interpreted as a strategy to optimize the body mass and thus to reduce the cost of flight. Indeed, low wing loading is advantageous for very slow flight and hovering (Witter and Cuthill, 1993). Barn Owls are utilizing such behaviours frequently during hunting (Taylor, 1994) and thus may be particularly penalized by the increase in energetic costs associated to the increased body mass. Likewise, Barn Owls catch prey up to $2 \mathrm{~km}$ from the nest site located in house garrets or roofs (Taylor, 1994). They must carry their prey from the hunting ground and over the surrounding houses to reach their nest, adding to the cost. Using an allometric relation between body mass and power necessary to fly, we show that there is indeed a significant energy saving by feeding after the hunting period. This behaviour was also observed in passerines that often feed their young most intensely early in the day (e.g. House Sparrow Passer domesticus (O'Connor, 1984) that is consistent with the view that the parents are reducing the impact of mass-dependent costs by foraging most intensely when their body mass is low.

The Barn Owl is specialised towards hunting micro-mammals such as field mice (Apodemus spp) and voles (Microtus spp) (Durant et al., 2010; Frey et al., 2011; Mikkola, 1983). However, the Barn Owl is also an opportunist which bases its hunting on the profitability of the prey (Ille, 1991) and captures species according to their availability. In nature, the period of hunt for the barn owl depends on the diel cycle of their prey. In eastern France, the main prey composing the diet of Barn Owl is the common vole (Microtus arvalis, (Durant et al., 2010). For microtine rodents, the most prominent activity peaks of the day commonly occur at dusk and dawn (see Halle, 1993). During reproduction, the barn owl hunts the most at the beginning of the night (Taylor, 1994). The advantage of eating at the end of the night is then double. It allows it to concentrate on hunting when food is highly available, and to do it with a lower flying cost 
(reduced wing loading, Norberg, 1996). Reduction of wind loading has already been hypothesized as a mechanism used to reduce the cost of foraging (Sanz and Moreno, 1995) during breeding, notably for the female (Durant et al., 2004). However, this hypothesis has been used to explain the body mass change during the entire reproduction period due to a spontaneous decrease of the food consumption. Here we show, as suggested for passerines (Thomas, 2000), that this hypothesis can be used to explain the diel regulation of body mass. Many previous studies have found that the body mass of birds shows a diel cycle with minima at the beginning of the activity period and maxima at the end (e.g. Blem, 1976; Evans, 1969; Haftorn, 1989, 1992; Kontgiannis, 1967; Stuebe and Ketterson, 1982; Thomas, 2000). Our study supports the idea that birds attempt to regulate their body reserves within a single day. This result is of interest as it provides evidence for the first time of how small raptors are strategically regulating their food intake to match the energy requirement and mass-dependent costs. Our results provides another example that the reduction of the body mass is not necessary linked to a cost or a negative energy budget. In our study the body mass decrease seemed to be linked to an optimisation of the energy budget and not to an increase of the male Barn Owl's energy requirement.

\section{Acknowledgments: This study was supported financially by the French Ministère de}

l'Environnement, Service de la Recherche des Etudes et du Traitement de l'Information sur l'Environnement, France. The experiments were done in compliance with current French laws and after acceptance by French authorities (authorisation of the Ministère de l'Agriculture et de la Pêche $n^{\circ}$ 04196). We thank C. Plumeré and J.-P Gendner for her help in developing the automatic nest, and M. Bertrand and B. Bertrand for the maintenance of the system. We are grateful to J. 
Durant, Hjermann \& Handrich

286 Lage of Jensen Software Systems (http://jensen-software.com) for his computer expertise. JMD and D.Ø.H thank CEES/University of Oslo for support.

288

289 Electronic supplementary material. The online version of this article contains supplementary

290 material, which is available to authorized users.

291 
Durant, Hjermann \& Handrich

\section{References}

Baudvin H (1980) Les surplus de proies au site de nid chez la chouette effraie (Tyto alba). Nos Oiseaux 35:232-238

Blem CR (1976) Patterns of lipid storage and utilization in birds. Am Zool 16:671-684

Bunn DS, Warburton AB, Wilson RDS (1982) The Barn Owl. T \& AD Poyser, Carlton, UK

Cade BS, Noon BR (2003) A gentle introduction to quantile regression for ecologists. Front Ecol Environ 1:412-420

Durant J (2000) Energétique de la reproduction chez la Chouette effraie (Tyto alba). University Louis Pasteur, Strasbourg

Durant JM (2002) The influence of hatching order on the thermoregulatory behaviour of barn owl Tyto alba nestlings. Avian Science 2:167-173

Durant JM, Gendner JP, Handrich Y (2004) Should I brood or should I hunt: a female barn owl's dilemma. Canadian Journal of Zoology-Revue Canadienne de Zoologie 82:1011-1016

Durant JM, Gendner JP, Handrich Y (2010) Behavioural and body mass changes before egg laying in the Barn Owl: cues for clutch size determination? J Ornithol 151:11-17

Evans PR (1969) Winter fat deposition and overnight survival of Yellow Buntings (Emberiza citrinella L.). J Anim Ecol 38:415-423

Frey C, Sonnay C, Dreiss A, Roulin A (2011) Habitat, breeding performance, diet and individual age in Swiss Barn Owls (Tyto alba). J Ornithol 152:279-290

Haftorn S (1989) Seasonal and diurnal body weight variations in titmice, based on analyses of individual birds. Wilson Bull 101:217-235

Haftorn S (1992) The diurnal body-weight cycle in titmice Parus spp. Ornis Scand 23:435-443 
Durant, Hjermann \& Handrich

314 Hakkarainen H, Koivunen V, Korpimäki E (1997) Reproductive success and parental effort of Tengmalm's owls: Effects of spatial and temporal variation in habitat quality. Ecoscience 4:35-42

Halle S (1993) Diel pattern of predation risk in microtine rodents. Oikos 68:510-518

Handrich Y, Nicolas L, Le Maho Y (1993) Winter starvation in captive common barn-owls Bioenergetics during refeeding. Auk 110:470-480

Ille R (1991) Preference of prey size and profitability in Barn owls Tyto alba guttata. Behaviour $116: 180-189$

Kontgiannis JE (1967) Day and night changes in body weight of the white-throated sparrow, Zonotrichia albicollis. Auk 84:390-395

Masman D, Gordijn M, Daan S, Dijkstra C (1986) Ecological energetics of the Kestrel: field estimates of energy intake throughout the year. Ardea 74:24-39

Maurer BA (1996) Energetics of avian foraging. In: Carey C (ed) Avian energetics and nutritional ecology. Chapman and Hall, New York, pp 250-279

Michelat D, Giraudoux P (1992) Activité nocturne et stratégie de recherhce de nourriture de la chouette effraie (Tyto alba) à partir du site de nidification. Alauda 60:3-8

Mikkola H (1983) Owls of Europe. Poyser, T//Poyser, AD, Carlton, UK

Muller Y (1991) La Chouette effraie (Tyto alba) dans la Réserve de la Biosphère des Vosges du Nord: fluctuations de populations, reproduction et régime alimentaire. Ann Sci Rés Bios Vosges du Nord 1:91-106

Mysterud A (2000) Diet overlap among ruminants in Fennoscandia. Oecologia 124:130-137

Newton I (1979) Population Ecology of Raptors. T \& AD Poyser Ltd, London 
Durant, Hjermann \& Handrich

Norberg UM (1996) Energetics of flight. In: Carey C (ed) Avian energetics and nutritional ecology. Chapman and Hall, New York, pp 199-249

O'Connor RJ (1984) The growth and development of birds. John Wiley, Chichester

R Development Core Team (2011) R: A language and environment for statistical computing. R Foundation for Statistical Computing, Vienna, Austria:URL http://www.R-project.org Rijnsdorp AD, Daan S, Dijkstra C (1981) Hunting in the Kestrel, Falco tinnunculus, and the adaptative significance of daily habits. Oecologia 50:391-406

Ritter F, Görner M (1975) Untersuchungen zur Aktivität und Ernährung der Schleiereule (Tyto alba) in Thüringen. Falke 24:344-348

Roulin A (2001) Food supply differentially affects sibling negotiation and competition in the barn owl (Tyto alba). Behav Ecol Sociobiol 49:514-519

Roulin A (2002) Offspring desertion by double-brooded female ban owls (Tyto alba). The Auk 119:515-519

Roulin A. 2004. The function of food stores in bird nests: observations and experiments in the barn owl Tyto alba. Ardea 92, 69-78

Sanz JJ, Moreno J (1995) Experimentaly induced clutch size enlargements affect reproductive success in the Pied Flycatcher. Oecologia 103:358-364

Schoener TW (1968) Anolis lizards of Bimini - Resource partitioning in a complex fauna. Ecology 49:704-726

Shawyer C (1994) The Barn Owl, Hamlyn species guides, London

Stearns SC (1992) The evolution of life histories. Oxford University Press, Oxford UK

Stuebe MM, Ketterson ED (1982) A study of fasting in tree sparrows (Spizella arborea) and dark-eyed juncos (Junco hyemalis) - Ecological implications. Auk 99:299-308 
Durant, Hjermann \& Handrich

Taylor I (1994) Barn Owls: Predator-prey relationships and conservation, Cambridge, University Press

Thomas RJ (2000) Strategic diel regulation of body mass in European robins. Anim Behav 59:787-791

Thouzeau C, Robin J-P, Le Maho Y, Handrich Y (1999) Body reserve dynamics and energetics of barn owls during fasting in the cold. J Comp Physiol B 169:612-620

Wijnandts H (1984) Ecological energetics of the Long-eared Owl (Asio otus). Ardea 72:1-92

Williams GC (1966) Natural selection, the costs of reproduction, and a refinement of Lack's principle. Am Nat 100:687-690

Witter MS, Cuthill IC (1993) The ecological costs of avian fat storage. Philos Trans R Soc Lond B 340:73-92

Wood SN, Augustin NH (2002) GAMs with integrated model selection using penalized regression splines and applications to environmental modelling. Ecol Model 157:157-177 


\section{FIGURE LEGENDS}

Fig. 1. Mean daily number of entries of six Barn Owl (Tyto alba) breeding pairs to the nest (males are filled triangles connected by solid line while females are open dots connected by dotted line). The shade area corresponds to the period used for the analysis. The vertical error bars are \pm SE and horizontal ones \pm SD.

Fig. 2. Body mass gain of male Barn Owls during the night. Each dot represents the increase of mass for a male measured between two weightings and is assumed to correspond to the food intake during the same period. The fitted nonparametric quantile regression every 0.1 quantile is presented in lines. The thick line is the 0.5 quantile. Lines over the 0.5 quantile are quantile 0.6 , 0.7, 0.8 and 0.9 and under the 0.5 quantile are quantile $0.4,0.3,0.2$ and 0.1 .

Fig. 3. Density plots displaying the males feeding habit (a. time when the heaviest meal of the day (max dBM) is more frequently taken) and prey delivery (b. the time when the heaviest prey of the day is more frequently brought to the nest) throughout the breeding season. Lighter is the colour (i.e., white) higher is the frequency of the event. The 2 hacked areas are daytime. a. The male behaviour seemed to change 2 times, first around hatching of the first egg (first dotted vertical line), and second at the female first exit (second dotted vertical line). b. The heaviest prey of a day are more often brought to the nest at the beginning of the night (hence caught at this time). After the first hatching (day 0) some heavy prey are brought at the end of the night. 
Figure 1

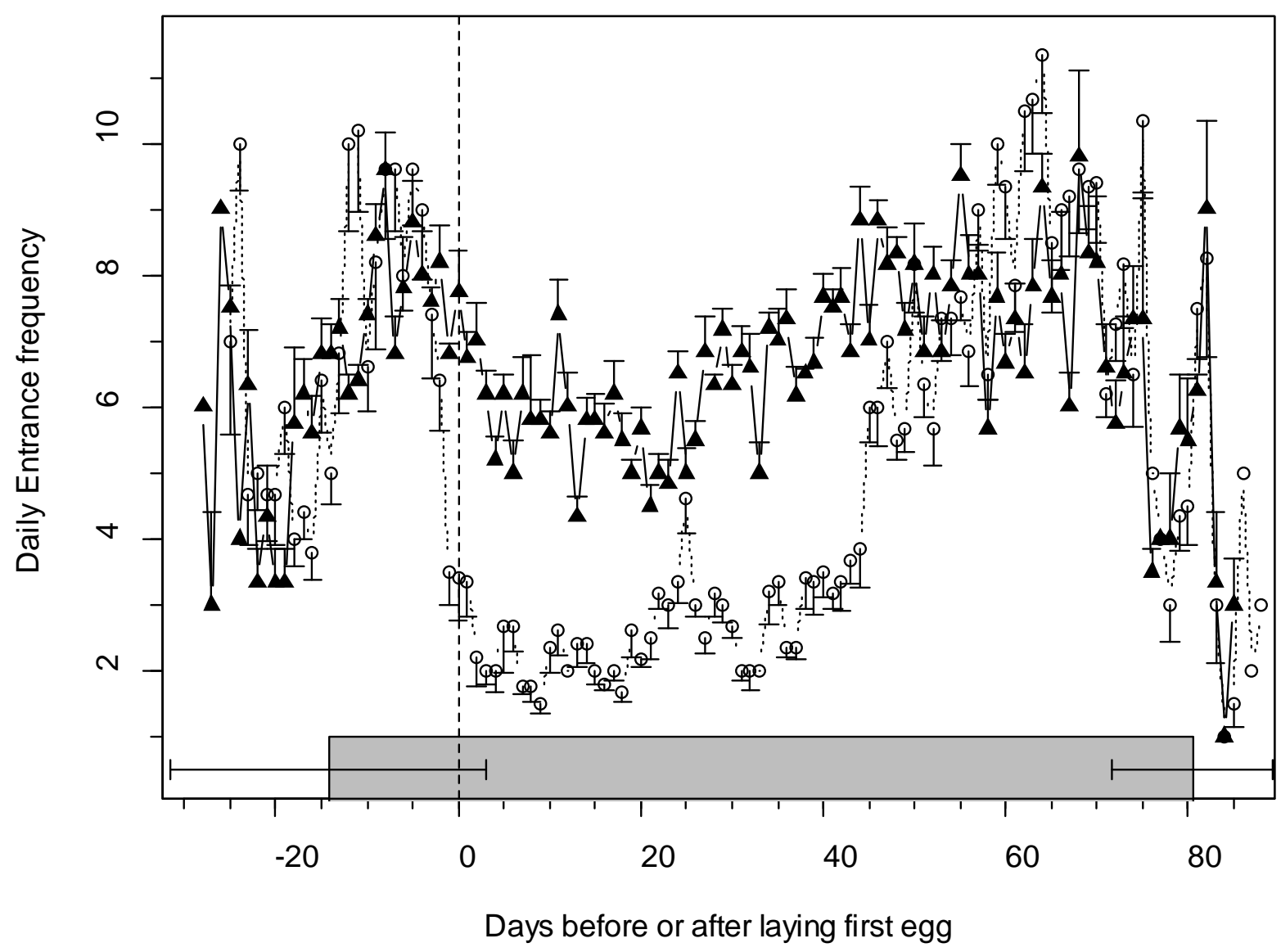


Durant, Hjermann \& Handrich

Figure 2

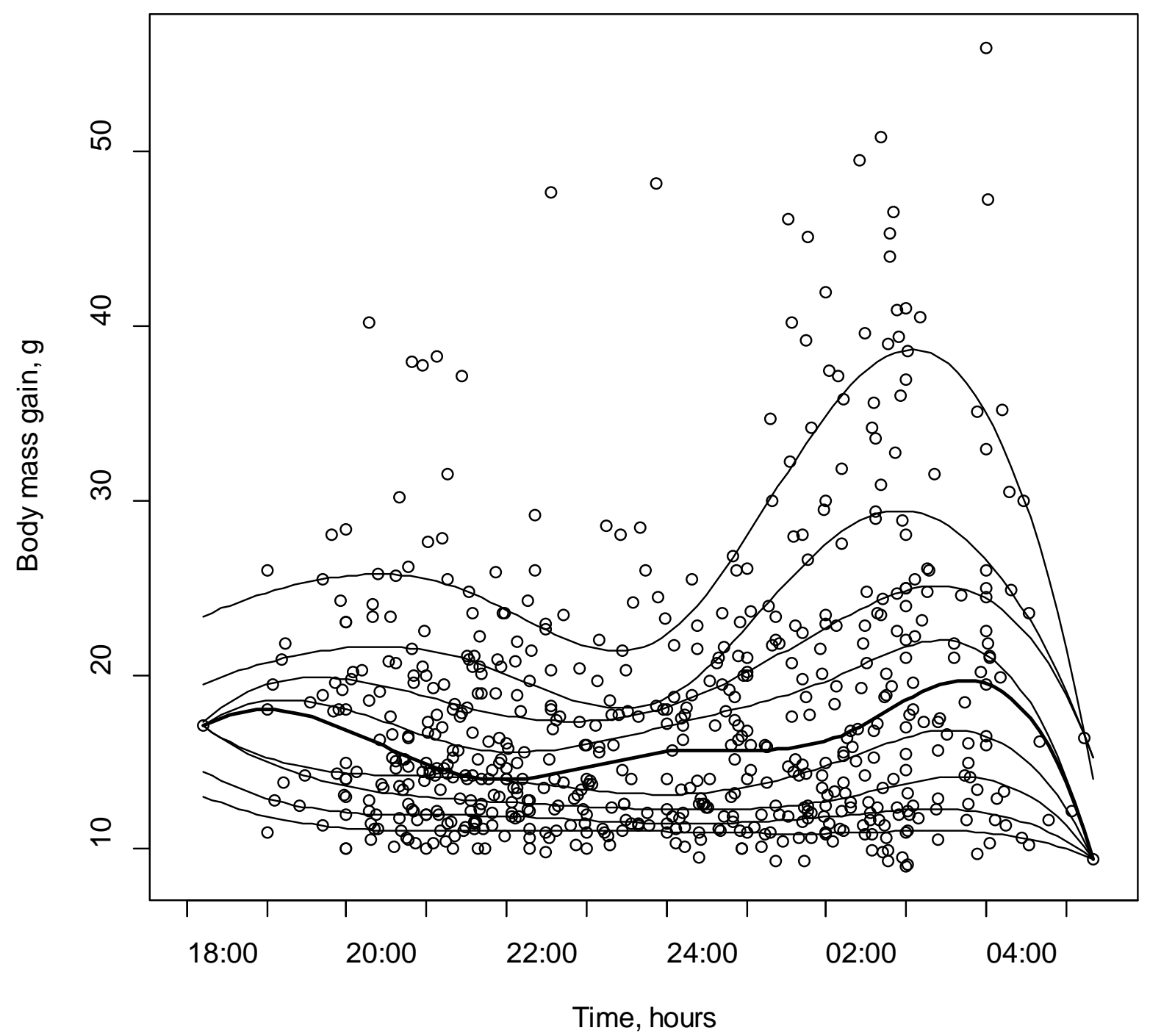

397 
Durant, Hjermann \& Handrich

$399 \quad$ Figure 3
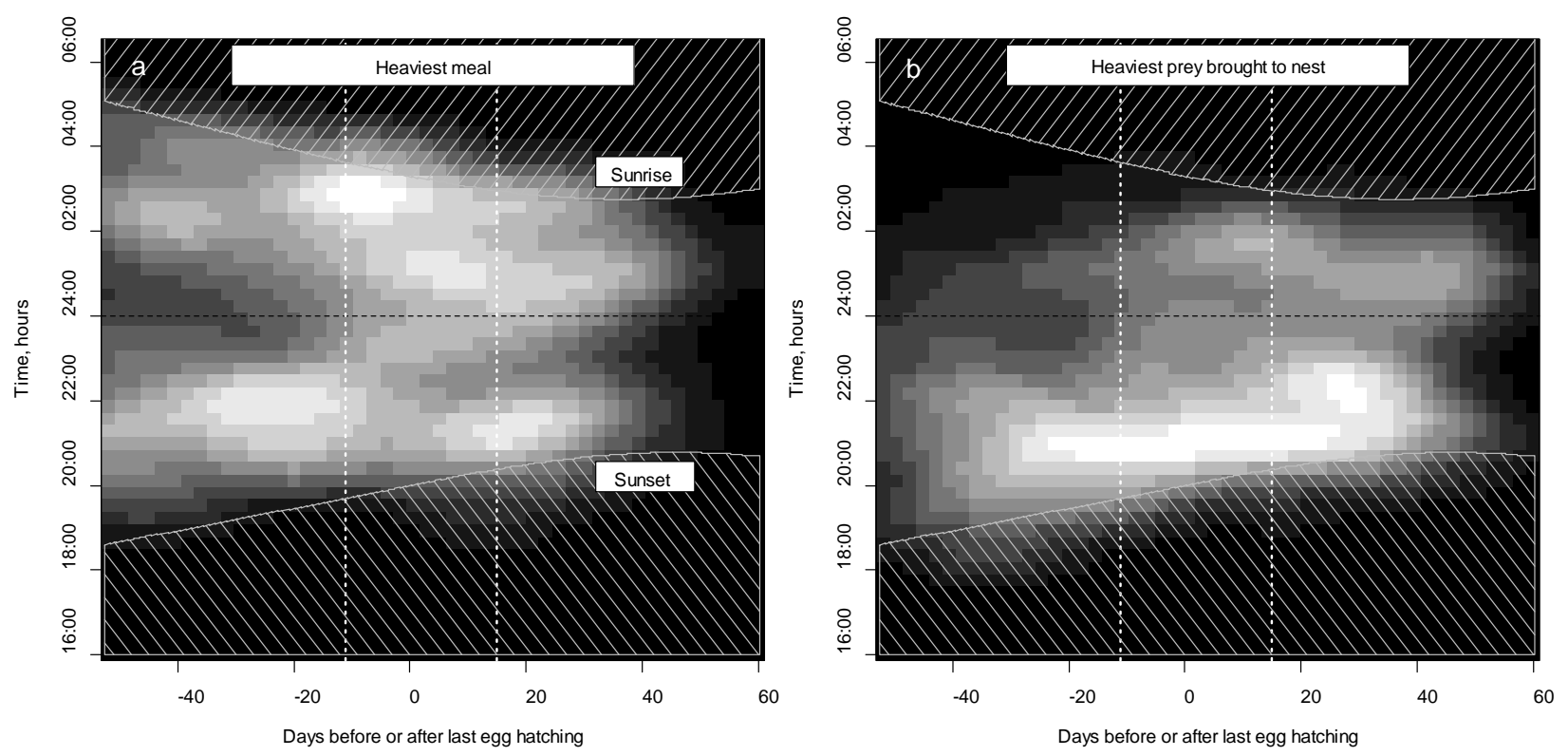

400

401 
Durant, Hjermann \& Handrich

402

403

404

405

406

407

408

409

410

411

412

413

\section{SUPPLEMENTARY MATERIAL}

\section{Diel feeding strategy during breeding in male Barn Owls (Tyto alba)}

Joël M. Durant, Dag Ø. Hjermann and Yves Handrich

\section{Supplementary figure 4: Body mass gain of male Barn Owls}
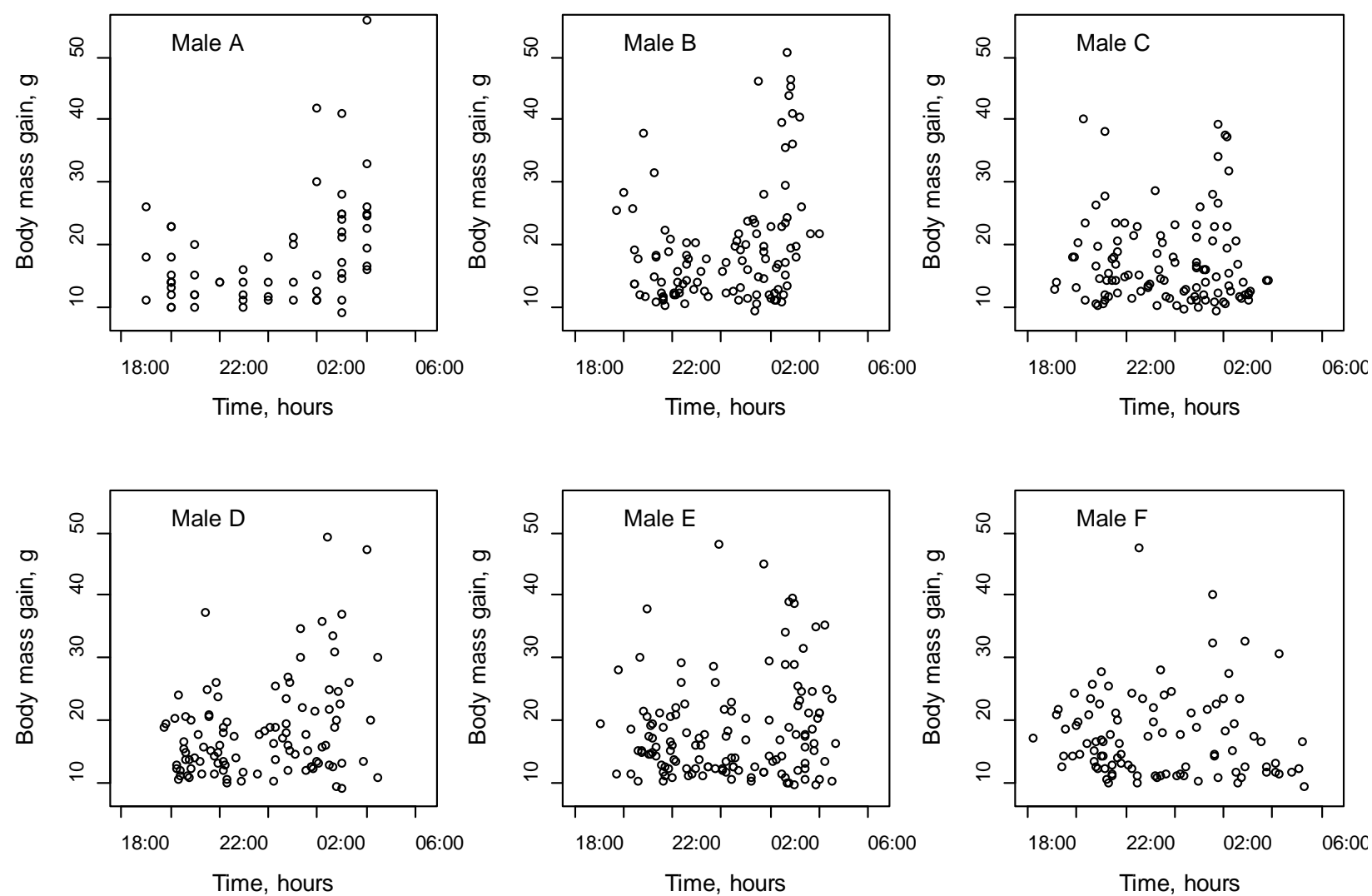

Figure 4. Body mass gains of male Barn Owls during the night. Each dot represents the increase of mass for a male measured between two weightings and is assumed to correspond to the food intake during the same period. 


\section{Supplementary figure 5: quantile regression analysis}

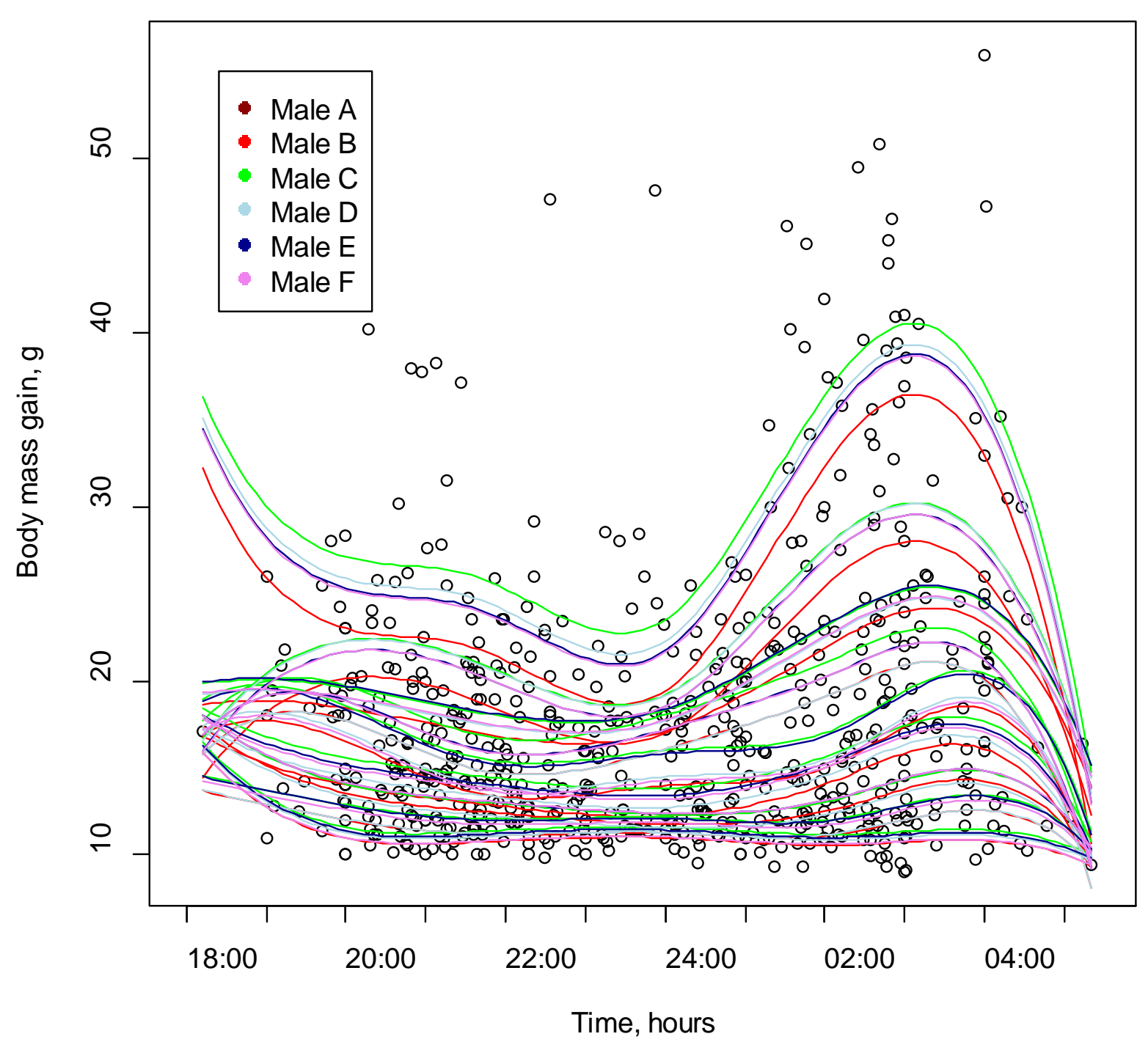

Figure 5. Body mass gain of male Barn Owls during the night. Each dot represents the increase of mass for a male measured between two weightings and is assumed to correspond to the food intake during the same period. The fitted nonparametric quantile regression with Male as factor variable every 0.1 quantile is presented in lines. 


\section{Supplementary figure 6: prey delivery analysis}
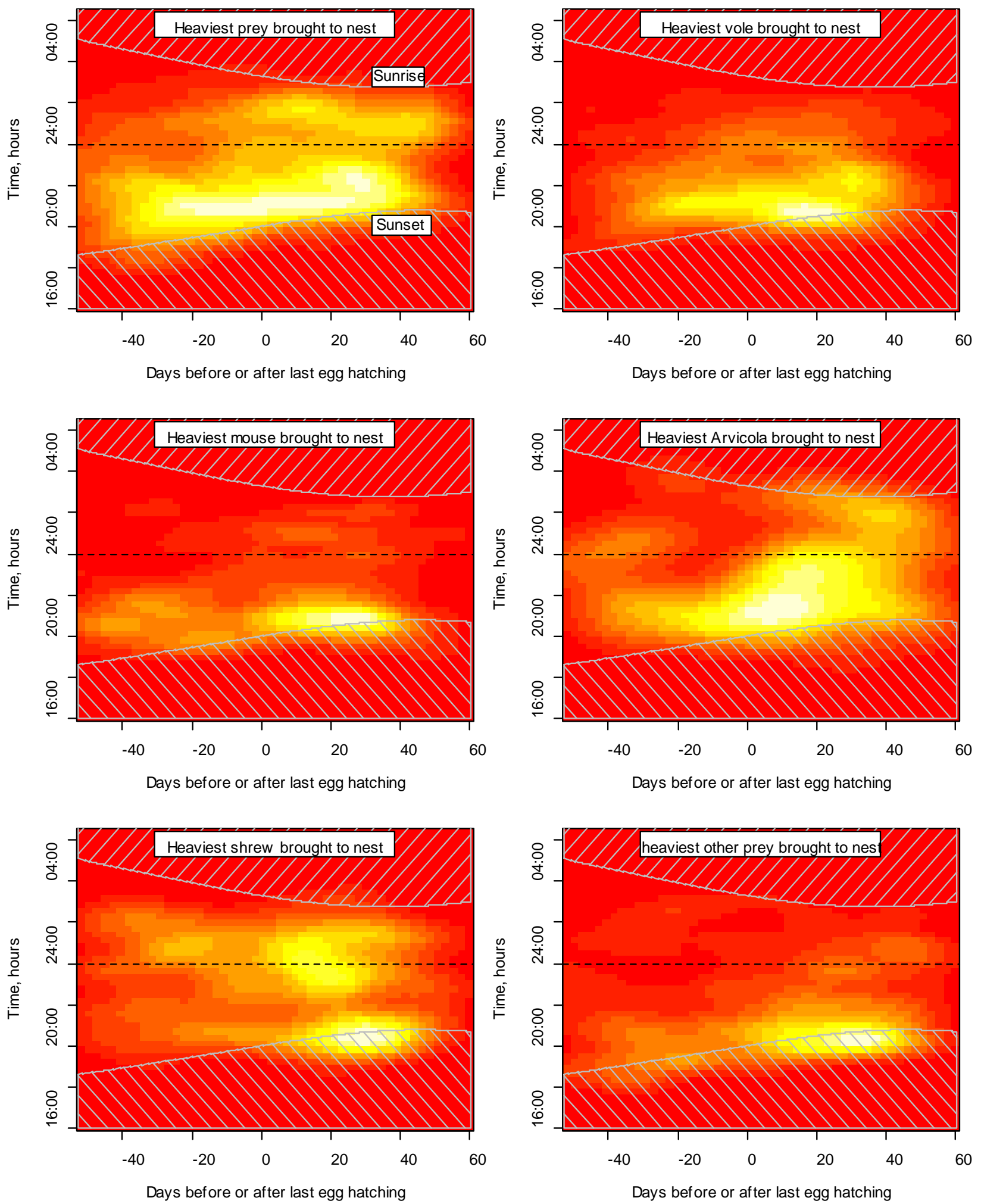

Figure 6. Density plots displaying prey delivery to the nest throughout the breeding season. Each 425 plot presents the time when the heaviest prey of the day is more frequently delivered to the nest. 
426 The density range from low (red, no event) to high frequency (yellow, many events). The 2

427 hacked areas are daytime.

428 The plot on the top left is a colour version of the Fig. 2a, i.e., all prey confounded. The heaviest

429 prey of a day are more often brought to the nest at the beginning of the night (hence caught at this

430 time). After the first hatching (day 0) some heavy prey are brought at the end of the night

431 corresponding to Arvicola (the heaviest species of the Barn Owl diet). 